\title{
LOS NIVELES DE VIDA EN LA ESPAÑA DEL SIGLO XVIII
}

\section{The standard of living in eighteenth-century Spain}

\author{
Héctor GARCÍA-MONTERO \\ Universidad Pública de Navarra \\ hector.garcia@unavarra.es
}

Fecha de recepción: 3/07/2018

Fecha de aceptación definitiva: 7/03/2019

RESUMEN: El trabajo plantea un estado de la cuestión sobre la bibliografía que ha abordado el estudio de los niveles de vida en la España del siglo XVIII. Al mismo tiempo se sugieren posibles vías de investigación para el futuro. El texto se organiza en relación a tres tipos de indicadores: de bienestar material y desigualdad económica (PIB por habitante, salarios reales e índice de Gini), biológico-nutricionales (estatura media) y demográficos (mortalidad y esperanza de vida). La evidencia disponible apunta a un balance ligeramente positivo en términos de bienestar material, matizado por un incremento de la desigualdad económica en la segunda mitad de la centuria, una caída del estatus nutricional entre los nacidos en las últimas décadas del siglo y una caída de la tasa de mortalidad bruta en la segunda mitad guiada por el descenso en la mortalidad ordinaria y adulta.

Palabras clave: España; siglo XVIII; nivel de vida; salarios; desigualdad; estatura; mortalidad.

ABSTRACT: This work provides a literature review of the research which dealt with the analysis of living standards in 18th-century Spain. At the same time, possible fields of research are suggested for the future. The text focuses on three types of indicators: material well-being and economic inequality (GDP per capita, real wages and Gini index), bio-nutritional (average height) and demographic (mortality and life expectancy) indicators. The available evidence points to a slightly positive balance in terms of material well-being, nuanced by an increase in economic inequality in the second half of the century, a drop in net nutritional status among those born 
in the final decades of the century, and a drop in mortality rates in the second half of the century, which was determined by a decline in ordinary and adult mortality.

Key words: Spain; 18th century; standard of living; wages; inequality; height; mortality.

\section{INTRODUCCIÓN}

El propósito principal de este artículo es trazar un estado de la cuestión sobre la historiografía que ha abordado el estudio de los niveles de vida en la España del siglo XVIII. La pertinencia de este trabajo se justifica por, al menos, tres razones principales. En primer lugar, no existe a día de hoy un estado de la cuestión que identifique los hallazgos, las carencias y las controversias de la historiografía ocupada en este tema y período, el cual, en definitiva, pueda ser de utilidad tanto a efectos ilustrativos y didácticos como para abordar futuras investigaciones. En segundo lugar, la historiografía española no se ha ocupado sino, en general, de forma tardía, dispersa e intermitente, del estudio de la evolución de las condiciones de vida ${ }^{1}$, por lo que el presente estado de la cuestión servirá para enumerar carencias y proponer vías por las que la investigación podría discurrir en el futuro. Finalmente, en tercer lugar, se espera que las propuestas de investigación sirvan en el futuro, más allá de su interés para la historiografía nacional, para una mejor inserción del caso español en el marco general de los debates sobre la coyuntura y las transformaciones socioeconómicas que vivió Europa durante el Siglo de las Luces.

El planteamiento general que guía este trabajo parte de una concepción plural del nivel o niveles de vida ${ }^{2}$ en la que juegan un papel fundamental indicadores que abordan aspectos diferentes a los estrictamente pecuniarios y a la medición de la desigualdad económica, como por ejemplo aquellos relacionados con la salud y la nutrición. Una concepción que se deriva de los planteamientos de autores como Amartya Sen, con su distinción entre funcionamientos y capacidades,

1. Véanse García-Montero, Héctor. «Los niveles de vida en la España del Antiguo Régimen. Estado de la cuestión y propuestas de investigación». En CHASTAGNARET, Gerard; DAumas, Jean-Claude; Escudero, Antonio y Raveux, Olivier (eds.). Los niveles de vida en España y Francia (siglos XVIIII-XX). Alicante: Publicaciones de la Universidad de Alicante-Publications de la Universitè de Provence, 2010, pp. 21-44 (p. 23). MARTínEZ-CARRIÓn, José Miguel. "El nivel de vida en la España rural, siglos XVIII-XX. Nuevos enfoques, nuevos resultados». En MARTínez-CARrión, José Miguel (ed.). El nivel de vida en la España rural, siglos XVIII-XX. Alicante: Publicaciones de la Universidad de Alicante, 2002, pp. 16-74 (p. 26). Yun, Bartolomé. "Introducción». En Yun, Bartolomé y Torras Elías, Jaume (dirs.). Consumo, condiciones de vida y comercialización: Cataluña, Castilla, siglos XVII-XIX. Valladolid: Consejería de Educación y Cultura-Junta de Castilla y León, 1999, pp. 1-26 (p. 7).

2. En aras de una mayor simplicidad, en este trabajo se emplearán como sinónimos términos como bienestar, condiciones de vida, nivel de vida, niveles de vida o calidad de vida. 
y que ha sido progresivamente reconocida y aceptada en las últimas décadas por agencias internacionales como el PNUD.

Al mismo tiempo, cabe señalar que, debido a razones de espacio, la necesaria concisión aconseja dejar al margen de este artículo aspectos relacionados con la medición de los niveles de vida tales como el funcionamiento de los mercados de trabajo o de la tierra, el consumo, la historia de las mujeres o de la infancia, la alfabetización o el capital humano. Aspectos, por otra parte, con enjundia más que suficiente como para recibir atención monográfica.

El artículo se organiza de la siguiente forma. Tras esta breve introducción, el epígrafe dos se ocupa del estudio de la evolución de los grandes agregados económicos como el PIB por habitante, los salarios e ingresos y la desigualdad económica. El tercer epígrafe se dedica a la historiografía ocupada de aspectos biológicos-nutricionales. El cuarto a las variables demográficas y, finalmente, en el quinto se esbozan unas breves conclusiones.

\section{CRECIMIENTO ECONÓMICO Y BIENESTAR MATERIAL}

El primer grupo de trabajos analizado es el de aquellos ocupados en medir los aspectos pecuniarios del bienestar. Más en concreto, en este epígrafe me ocuparé, en primer lugar, de la revisión de la historiografía que ha tratado de estimar el PIB por habitante o magnitudes relacionadas; posteriormente, de aquella otra ocupada en evaluar la trayectoria de los salarios y su poder adquisitivo y de tratar de aproximar los ingresos personales y/o familiares, y, finalmente, de la literatura dedicada al estudio de la desigualdad económica.

\subsection{El PIB por habitante}

Desde el punto de vista del desempeño económico, la visión tradicional moderna de la España del Setecientos, cuyas raíces pueden remontarse al clásico de Gonzalo Anes Las crisis agrarias en la España moderna ${ }^{3}$, marca una discontinuidad clara entre el medio siglo transcurrido tras la Guerra de Sucesión y una segunda fase, con un límite temporal más difuso, que puede situarse entre los últimos años de la centuria y la crisis de subsistencias de los años 1802-1805 o la Guerra de la Independencia.

Mientras en la primera etapa tuvo lugar una expansión generalizada, en la que la mayor parte de los territorios de la periferia peninsular superaron ampliamente las cotas máximas de producción agraria de fines del siglo XVI y en la España interior se recuperó buena parte o todo el terreno perdido -llegándose incluso a superar en algunos casos-, en la segunda, el agotamiento del modelo

3. ANES, Gonzalo. Las crisis agrarias en la España moderna. Madrid: Taurus, 1970. 
de crecimiento agrario, básicamente extensivo y sujeto, por tanto, a la dialéctica maltusiana entre población y recursos, habría provocado una desaceleración y un aumento de las fluctuaciones hasta llegar a la parálisis o incluso la depresión; con su punto culminante en la gran crisis de 1802-1805. No obstante las crecientes y significativas diferencias estructurales entre los tres grandes modelos de desarrollo económico, el de la España húmeda galaico-cantábrica, el mediterráneo y el de las "Españas interiores», este diagnóstico se ha aplicado a muy grandes rasgos, con causas parcialmente distintas y con todos los matices que se quiera, a todas las zonas; aunque de forma especialmente pertinente al interior.

A pesar de las numerosas cautelas y espacios de sombra que el propio Anes reconoció en lo referente al último período del Setecientos ${ }^{4}$ y de las casi cinco décadas transcurridas desde la aparición de Las Crisis, este esquema ha sido revalidado en numerosos trabajos, perdurando hasta tiempos recientes con notable salud y vigencia. Empero, mientras en lo relativo a las décadas inmediatamente posteriores a la Guerra de Sucesión dicho planteamiento ha ofrecido hasta ahora pocas fisuras, no sucede lo mismo con la perspectiva sombría sobre el último tramo del siglo.

En este sentido, una creciente literatura ${ }^{5}$ ha revisado la interpretación tradicional sobre el desempeño de la economía española, sobre todo de la economía de la España interior, en dicho período. En síntesis, la posición de estos autores es que la economía española a finales del siglo XVIII, lejos de alcanzar todo su potencial de crecimiento dadas las restricciones ambientales y tecnológicas de la época, era presa de límites institucionales y sociales -los estorbos morales y políticos de los que hablara Jovellanos- que constreñían su crecimiento, el cual, además, ha sido probablemente infravalorado. En este sentido, una de las principales críticas es la aparente incoherencia que supone el hecho de pensar en términos maltusianos -agotamiento de un modelo de crecimiento extensivo- cuando en la primera mitad del siglo xIX el sector agrario sería capaz de alimentar a una población mucho mayor sin mediar cambios profundos en el modelo productivo.

Desde un punto de vista cuantitativo, son relativamente recientes los intentos de estimar la trayectoria seguida por macromagnitudes económicas, como el PIB por habitante, en la España Moderna. En los últimos años y a raíz del debate planteado por la corriente historiográfica conocida como «la revuelta de los modernistas" (the revolt of the early modernists), la hipótesis de que las economías europeas fueron capaces en algunos casos de generar crecimiento económico en

4. ANES, Gonzalo. Las crisis..., op. cit., pp. 162-166.

5. Los ejemplos más relevantes, en los que se exponen el estado de la cuestión y las contribuciones de esta línea de pensamiento, serían los textos de Llopis, Enrique. "Expansión, reformismo y obstáculos al crecimiento (1715-1789)». En Comín, Francisco; HeRnándEZ, Mauro y Llopis, Enrique (eds.). Historia Económica de España. Siglos X-XX. Barcelona: Crítica, 2002, pp. 121-164 y SEBASTIÁN, José Antonio. "La agricultura española y el legado del Antiguo Régimen». En Llopis, Enrique (ed.). El legado económico del Antiguo Régimen en España. Barcelona: Crítica, 2004, pp. 147-186. 
el largo plazo durante la Edad moderna, diversos trabajos han acometido la tarea de «aventurarse» a dar cifras acerca de la evolución de las principales macromagnitudes económicas en la España Moderna. De este modo, en consonancia con una tendencia historiográfica seguida en otros países de Europa occidental, autores como Yun, Carreras y Álvarez Nogal y Prados de la Escosura han estimado, para la Corona de Castilla en el primer caso y para el conjunto de España en los otros, el PIB por habitante en el período 1500-1800. Por su parte autores como van Zanden, Allen, Maddison o Malanima han incluido en paneles internacionales sus propias estimaciones sobre el caso español ${ }^{6}$.

Una característica común de dichas estimaciones es haberse basado en una metodología que afronta el cálculo del PIB por habitante desde el lado de la demanda apoyándose en la estimación del consumo por habitante a partir de salarios de trabajadores urbanos -normalmente del sector de la construcción-, los precios de bienes agrarios y no agrarios y supuestos acerca del valor de la elasticidad precio y la renta de demanda. Una vez calculado el consumo por habitante, los datos y estimaciones sobre la trayectoria seguida por la población y el supuesto de saldos comerciales - positivos o negativos- marginales completan la ecuación que permite el cálculo del producto por habitante.

La valoración del crecimiento económico español durante el siglo XVIII que se desprende de estos estudios ha oscilado entre el moderado optimismo de las cifras de Carreras, según las cuales la economía española habría crecido a una tasa anual acumulativa del $0,32 \%$, pasando por las estimaciones también optimistas -una tasa de crecimiento de entre un 0,17 y un 0,28\%- de Yun para la Corona de Castilla durante la segunda mitad del siglo XviII, las de van Zanden para todo el siglo XVIII con un 0,23\%, las de Maddison - para el período comprendido entre 1700 y 1820 - de un $0,14 \%$ anual y el pesimismo de los primeros cálculos ofrecidos por Álvarez Nogal y Prados de la Escosura, según los cuales el PIB por habitante entre 1700 y 1787 en España se habría contraído a una tasa del -0,02\% anual.

Cabe señalar, sin embargo, que las estimaciones de Álvarez Nogal y Prados de la Escosura han sido reelaboradas con posterioridad ofreciendo una visión

6. Véanse: ALLEN, Robert C. «Economic structure and agriculture productivity in Europe, 13001800». European Review of Economic History, 2000, 3, 2, pp. 1-25. ÁlvarEZ-Nogal, Carlos y Prados DE LA EsCODURA, Leandro. "The decline of Spain (1500-1850): conjectural estimates». European Review of Economic History, 2007, 11, 3, pp. 319-366. Álvarez-Nogal, Carlos y Prados de la Escosura, Leandro. "The rise and fall of Spain (1270-1850)". Economic History Review, 2013, 66, 1, pp. 1-37. CARRERA, Albert. "Modern Spain". En MOKYr, Joel (ed.). The Oxford Encyclopedia of Economic History. 5 vols. New York: Oxford University Press, 2003, 4, pp. 546-553. Malanima, Paolo. "The Long Decline of a Leading Economy: GDP in Central and Northern Italy, 1300-1913». European Review of Economic History, 2011, 15, pp. 169-219. Yun, Bartolomé. "Proposals to quantify long-term performance in the Kingdom of Castile, 1550-1800». En Maddison, Angus y VAn Der WeE, Herman (eds.). Economic Growth and Structural Change: Comparative Approaches over the Long Run. Milano: Università Bocconi, 1994, pp. 97-110. VAN ZANDEN, Jan Luiten. "Una estimación del crecimiento económico en la Edad Moderna». Investigaciones de Historia Económica, 2005, 1, 2, pp. 9-38. 
menos sombría. Según las últimas cifras ofrecidas por estos autores la economía española habría crecido durante el Setecientos a una tasa del 0,11\% o 0,12\% anual $^{7}$. Un crecimiento sin duda modesto, pero positivo.

No obstante, en los últimos años, no han faltado voces -en no pocas ocasiones las de los mismos autores- alertando de los problemas y las limitaciones de la metodología de base que subyace en este tipo de estimaciones ${ }^{8}$. Limitaciones como suponer un número constante de días trabajados a lo largo del año durante centurias, asumir que las rentas del capital evolucionaron de forma similar a las del trabajo, la dependencia de las series salariales procedentes del sector de la construcción en ciertas ciudades importantes o los cambios en las dietas y en los patrones de consumo de productos agrarios.

No es extraño, por tanto, que se haya señalado la pertinencia de acometer las estimaciones del PIB -sobre todo de la producción agraria-, como ha comenzado a hacerse también para otros países europeos como Inglaterra u Holanda9, por la vía del producto y a partir de la recopilación de evidencia directa procedente de fuentes primarias. A este respecto cabe señalar el reciente trabajo de ÁlvarezNogal, Prados de la Escosura y Santiago-Caballero ${ }^{10}$, en el que el cálculo de la producción agraria a partir de la recopilación de series diezmales publicadas por diversos autores refuerza el panorama sombrío sobre el desempeño de la agricultura española durante la Edad Moderna. Un panorama que, en lo que atañe al Setecientos, apuntaría a un estancamiento o leve descenso del producto agrario por habitante y a un ligero incremento por activo empleado en la agricultura.

Dichos resultados, por otro lado, han sido cuestionados recientemente en diversos trabajos publicados por el Grupo Complutense de Historia Económica Moderna $^{11}$. Según estos autores, el balance de la evolución de la producción agraria quedaría ensombrecido al no tenerse en cuenta varios factores que distorsionan las series diezmales en las últimas décadas del siglo XVIII, sesgándolas sensible y crecientemente a la baja, y resulta difícil de explicar a la vista del auge demográfico generalizado que se detecta en los diferentes territorios peninsulares y del

7. Álvarez Nogal, Carlos y Prados de la Escosura, Leandro. "The rise and fall...», op. cit.

8. Véase el trabajo de Enrique Llopis y José Antonio Sebastián incluido en este mismo número monográfico.

9. Para Inglaterra véase Broadberry, Stephen; CAmpbell, Bruce; Klein, Alexander; Overton, Mark y LeEuwen, Bas van. British Economic Growth 1270-1850. Cambridge: Cambridge University Press, 2015; y para Holanda VAN Zanden, Jan Luiten y VAN LeEuwen, Bas. "Persistent but not consistent: the growth of national income in Holland, 1347-1807». Explorations in Economic History, 2012, 49, 2 , pp. 119-130.

10. Álvarez Nogal, Carlos; Prados de la Escosura, Leandro y Santiago-Caballero, Carlos. "Spanish agriculture in the little divergence». European Review of Economic History, 2016, 20, 4, pp. $452-477$.

11. No me detendré en detallar los argumentos de este grupo de investigadores ya que se desgranan en detalle en el trabajo de Enrique Llopis y José Antonio Sebastián incluido en este mismo monográfico. 
hallazgo de una reducción en la mortalidad -en especial la ordinaria y adulta- en ambas Castillas y en algunas regiones de la periferia peninsular (véase el epígrafe sobre indicadores demográficos de este trabajo).

En definitiva, aun quedando todavía un gran espacio para el debate y nuevas investigaciones, todo parece indicar que en el Siglo de las Luces las «luces» sobre la evolución del bienestar material han ido imponiéndose a las «tinieblas» de algunas estimaciones iniciales. Un panorama de ligero optimismo ${ }^{12}$ que contrasta con la visión sombría aún predominante sobre el desempeño de la economía española durante las centurias de la Edad Moderna. Asunto que, sin embargo, escapa a los objetivos y el alcance de este trabajo.

Empero, la defensa de un moderado optimismo por lo que toca al incremento del producto por habitante no implica trasladar directamente dicho aumento a una mejora generalizada de las condiciones de vida de la población; ni siquiera a las estrictamente materiales.

\subsection{Jornales, salarios e ingresos}

Durante décadas la "historia de los precios", en su versión de reconstrucción de series de jornales, salarios y precios, fue la tarea principal, cuando no única, de aquellos historiadores económicos interesados en el estudio de los niveles de vida. La relativa abundancia de datos, el interés en obtener un potencial indicador del poder adquisitivo de las clases populares, cierta sensibilidad intrínseca -a diferencia del PIB por habitante- a la desigualdad económica al centrarse en el trabajo asalariado y la posibilidad de realizar comparaciones del poder adquisitivo entre distintos grupos sociales o ámbitos geográficos explicarían las razones de su popularidad.

Ello no ha evitado, sin embargo, frecuentes críticas a una identificación demasiado simplista entre salario o jornal -casi siempre masculino- e ingreso personal o familiar. En este sentido, a los retos que plantea la construcción de índices de precios, se suman los relacionados con el desconocimiento de los datos necesarios para pasar del jornal o salario al ingreso personal o familiar: el porcentaje que representa la población asalariada sobre el total de la población activa, el número de días laborables y de horas trabajadas, el nivel de desempleo, los salarios percibidos en especie, los jornales femeninos o infantiles, los ingresos procedentes de ventas de la producción doméstica, la valoración de la apropiación de bienes

12. Un relativo optimismo que ya fue sugerido en RINGROSE, David. España, 1700-1900: el mito del fracaso. Madrid: Alianza Universidad, 1996, pp. 92-93. En dicha obra, desde una perspectiva muy general y plurisecular y a contracorriente de buena parte de la historiografía, el autor norteamericano defendió que, desde finales del siglo XVII o inicios del siglo XVIII, España, aun con diferencias regionales y altibajos puntuales motivados por conflictos bélicos o crisis de subsistencias, habría experimentado un lento y sostenido crecimiento del producto por habitante. 
libres y del disfrute de bienes públicos ${ }^{13}$, el autoconsumo e incluso el mero desconocimiento de las retribuciones de gran parte del "sector asalariado". Por tanto, el cálculo de series de jornales y salarios es un input esencial del estudio del ingreso, pero no el único. Un input que, en cualquier caso, debe tratar de situarse en las coordenadas de un determinado contexto socioeconómico e histórico y, preferiblemente, junto a otros indicadores de bienestar.

En el caso de la España Moderna, tras los tempranos y pioneros estudios de Hamilton, la historiografía española vivió una "larga siesta» en la que durante décadas apenas se volvió a interesar por el cálculo de series de salarios y precios para este periodo ${ }^{14}$. No en vano, las series de precios y salarios urbanos de Hamilton han sido utilizadas durante años -en bruto o reelaboradas-como referente e input básico por gran parte de la historiografía nacional e internacional ${ }^{15}$.

El patrón temporal seguido por las series de salarios reales urbanos a lo largo del Setecientos indica un estancamiento o leve crecimiento del poder adquisitivo durante la primera mitad de la centuria y una tendencia descendente desde mediados -y especialmente en las décadas finales- del Setecientos hasta un punto de inflexión en torno a los primeros años del Ochocientos. Un panorama que parecen corroborar los datos sobre el poder adquisitivo de los jornales agrícolas recopilados por Bringas ${ }^{16}$ para el conjunto del país en algunos años concretos de la segunda mitad de la centuria, apuntando a una caída generalizada de en torno a un tercio en términos reales.

A pesar del efecto intimidador que el enorme legado del historiador norteamericano parece haber ocasionado en la historiografía española, la historia de los precios parece haber adquirido nuevos bríos en los últimos tiempos. Una nueva

13. Sobre el impacto de la disponibilidad de bienes comunales en indicadores de bienestar como la esperanza de vida y la estatura media, véase BELTRÁn TAPIA, Francisco. "Commons and the standard of living debate in Spain». Cliometrica, 2015, 9, 1, pp. 27-48.

14. Quizás la excepción más notable sean los trabajos de Gaspar Feliu para la ciudad de Barcelona. Véanse Feliu, Gaspar. "Precios y Salarios en la Cataluña Moderna. Volumen I: alimentos». Estudios de Historia Económica, 1991a, 21; "Precios y Salarios en la Cataluña Moderna. Volumen II: Combustibles, productos manufacturados y salarios». Estudios de Historia Económica, 1991b, 22; y “Aproximació a un índex del cost de la vida a Barcelona, 1501-1807». En FonTANA, Josep (ed.). Història i projecte social. Reconeixement a una trajèctoria. Barcelona: Crítica, 2004, pp. 151-170.

15. Véanse, por ejemplo, Allen, Robert C. "The Great Divergence in European Wages and Prices from the Middle Ages to the First World War». Explorations in Economic History, 2001, 38, 4, pp. 411447. MarTín ACEÑA, Pablo. "Los precios en Europa durante los siglos XVI y XVII: estudio comparativo". Revista de Historia Económica-Joumal of Iberian and Latin American Economic History, 1992, 10, 3, pp. 359-395. ReHer, David Sven y Ballesteros Doncel, Esmeralda. "Precios y salarios en Castilla la Nueva: la construcción de un índice de salarios reales, 1501-1991". Revista de Historia EconómicaJournal of Iberian and Latin American Economic History, 1993, 11, 1, pp. 101-151. VAN ZANDEN, Jan Luiten. "Wages and the standard of living in Europe, 1500-1800». European Review of Economic History, 1999, 3, 2, pp. 175-197.

16. Bringas Gutiérrez, Miguel Ángel. La productividad de los factores en la agricultura española (1752-1935). Madrid: Banco de España, 2000, pp. 60 y 96. 
historia de los precios no tanto interesada ya en trazar las grandes tendencias seguidas por los salarios y su poder adquisitivo, sino, más allá de ampliar y mejorar la base factual, en afrontar el tema bajo horizontes metodológicos más específicos y sofisticados $^{17}$.

En primer lugar, se ha señalado la necesidad de seguir construyendo series de jornales y salarios. En particular para el ámbito rural, en el cual apenas disponemos de series que cubran toda la centuria ${ }^{18}$, mucho menos la Edad Moderna, y en el ámbito de ciudades pequeñas o de tamaño medio, hasta ahora infrarrepresentadas en los estudios sobre salarios y $\operatorname{precios}^{19}$. Cuestión no baladí si atendemos a las distorsiones introducidas por la fiscalidad y los privilegios del abastecimiento urbano en las grandes ciudades.

Por otro lado, en lo que atañe al ámbito urbano, la construcción de nuevas series salariales o la reelaboración de las ya existentes debería ampliar su espectro más allá del habitual de los trabajadores del sector de la construcción, incluyendo series para otros oficios industriales y/o artesanales, así como para empleados del sector servicios. En el mundo rural las series de jornales deberían calcularse y ordenarse según las distintas faenas agrícolas atendiendo a las características que determinaron la cuantía de los pagos: la época del año en que se realizaron y, por tanto, longitud de la jornada de trabajo, el grado de cualificación necesario para su ejecución y el sexo y la edad del trabajador ${ }^{20}$. Además, debe indagarse sobre las retribuciones de los criados, mozos de labranza o empleados fijos, las cuales

17. Véanse, entre los que cubren el siglo XVIII, CALDERón FERnÁndeZ, Andrés; García-MonTERo, Héctor y Llopis, Enrique. "New research guidelines for living standards, consumer baskets, and prices in Madrid and Mexico». En NIGRO, Gianpiero (ed.). I prezzi delle cose nell'eta preindustriale XLVIIISettimana di Studi Istituto Internazionale di Storia Economica "F. Datini». Firenze: Firenze University Press, 2017, pp 333-364. LóPEZ-Losa, Ernesto y PIquero-Zarauz, Santiago. "Spanish real wages in the North-western mirror, 1500-1800. On the timings and magnitude of the Little Divergence in Europe». Documento de Trabajo de la AEHE, 2016, n. ${ }^{\circ}$ 16-07. LloPIs, Enrique y GARCía-MonTERO, Héctor. "Precios y salarios en Madrid, 1680-1800». Investigaciones de Historia Económica, 2011, 7, 2, pp. $295-309$.

18. Algunas series, aun nacidas con una clara vocación contemporánea, tienen la virtud de arrancar en distintos momentos a lo largo del siglo XVIII. Véanse para Navarra LANA BERASAIN, José Miguel. «El poder de compra de jornaleros y criados: Salarios reales y mercados de trabajo en la Navarra rural (1781-1936)». Investigaciones de Historia Económica, 2007, 7, 1, pp. 37-68. Para Cataluña Garrabou, Ramón y Tello, Enric. "Salario como coste, salario como ingreso: el precio de los jornales agrícolas en la Cataluña contemporánea, 1727-1930». En MARTínEZ-CARRIón, José Miguel (coord.). El nivel de vida..., op. cit., pp. 113-182. Para la ciudad de Palencia y sus alrededores MORENO LÁZARO, Javier. "¿Fomentó el capitalismo agrario la desigualdad? Salarios y niveles de vida en Castilla la Vieja, 1751-1861». En MarTínez-Carrión, José Miguel (coord.). El nivel de vida ..., op. cit., pp. 16-74.

19. Véase un estudio reciente sobre la localidad soriana de El Burgo de Osma en PÉrEz ROMERO, Emilio. "Precios, salarios y carga laboral durante el siglo XVIII. El caso de la villa de El Burgo de Osma (Soria)». Investigaciones de Historia Económica-Economic History Research, 2019, 15, 2, pp. 78-90.

20. García-Montero, Héctor. "Historia agraria y niveles de vida. Estado de la cuestión y propuestas de investigación para la España contemporánea». En SOTO FERNÁNDEZ, David y LANA BERASÁIN, José Miguel (eds.). Del pasado al futuro como problema. La bistoria agraria contemporánea española en el siglo XXI. Zaragoza: Prensas Universitarias de Zaragoza, 2019, pp. 125-144 (pp. 130-131). 
solían pagarse en forma de salarios anuales o mensuales, y sobre las faenas realizadas a destajo. Asimismo, es necesario computar las retribuciones en especie, no siempre conocidas ni fáciles de calcular, pero especialmente importantes en el caso de los criados $^{21}$.

En tercer lugar, es indispensable incrementar la base factual de series con los pagos a trabajos realizados por mujeres y menores de edad, los cuales permiten añadir nuevos ingredientes a la ecuación de los ingresos familiares y calcular la brecha salarial en pagos por la misma o similar faena (el gender gap) y su evolución comparada entre regiones y en el contexto internacional ${ }^{22}$. Máxime si, como ha demostrado un trabajo reciente de Carmen Sarasúa basado en declaraciones de familias de la España interior rural en el Catastro de Ensenada ${ }^{23}$, la tasa de actividad femenina fue significativamente más alta de lo que tradicionalmente se había pensado; en especial en el sector manufacturero.

La estimación del número de días trabajados y su evolución es otro de los ingredientes básicos del cálculo de los ingresos familiares, pudiendo llegar a determinar la supervivencia del individuo o la unidad familiar, dependiente tanto del valor de los pagos recibidos por jornal como del monto total de días efectivamente trabajados ${ }^{24}$.

21. Ibid., p. 130 .

22. A este respecto, entre los centrados específicamente en dicha temática y en el siglo XVIII, BORRAS LLOP, José María. "Una historia recuperada. Las aportaciones de la infancia al crecimiento económico y a la subsistencia familiar». En Borras Llop, José María (coord.). El trabajo infantil en España, (1750-1950). Barcelona: Icaria y Publicaciones y Edicions de la Universitat de Barcelona, 2013, pp. 9-26. HeRnández García, Ricardo. "El trabajo en la industria textil de Tierra de Campos a mediados del siglo XVIII: la fábrica de Astudillo». En SARASÚA, Carmen y GÁLVEZ, Lina (eds.). ¿Privilegios o eficiencia?: Mujeres y hombres en los mercados de trabajo. Alicante: Publicaciones de la Universidad de Alicante, 2003, pp. 113-135. HeRnáNDEZ García, Ricardo. "La mano de obra infantil en la Castilla rural del siglo XVIII: El trabajo del niño es poco pero el que lo desprecia, un loco». En BorRas Llop, José María (coord.). El trabajo infantil..., op. cit., pp. 91-116. Jover AVELLÀ, Gabriel. "La difusión del trabajo asalariado infantil en la agricultura mallorquina: una aproximación a partir de los libros de cuentas de los predios oleícolas, 1700-1850». En Borras Llop, José María (coord.). El trabajo infantil..., op. cit., pp. 27-62; y "Salarios y movilización del trabajo femenino en el olivar: Mallorca, 1650-1720». En OrTEGA LóPEZ, María Teresa (ed.). Jornaleras, campesinas y agricultoras: la historia agraria desde una perspectiva de género. Zaragoza: Prensas Universitarias de Zaragoza, 2015, pp. 43-64. SARASÚA, Carmen. "La industria del encaje en el campo de Calatrava". Arenal: Revista de Historia de las Mujeres, 1995, 2, 2, pp. 151-174; "El oficio más molesto, más duro: el trabajo de las lavanderas en la España de los siglos XVIII al XX". Historia Social, 2003, 45, pp. 53-78; y "AActivos desde cuándo? La edad de acceso al mercado de trabajo en la España del siglo XVIII». En Borras Llop, José María (coord.). El trabajo infantil..., op. cit., pp. 63-90.

23. SARASÚA, Carmen. "Women's work and structural change: occupational structure in eighteenth-century Spain». Economic History Review, 2019, 72, 2, pp. 481-509.

24. Véanse García ZúÑIGa, Mario. "Fêtes chômées et temps de travail en Espagne (1250-1900)». En MaITTE, Corine y Terrier, Didier (dirs.). Les temps du travail. Normes, pratiques, évolutions (XIVe-XIXe siècles). Rennes: Presses Universitaires de Rennes, 2014, pp. 63-80. GARCÍA-ZÚÑIGa, Mario y LóPEZ-Losa, Ernesto. "Building Workers in Madrid (1737-1805). New Wage Series and Working Lives». EHES Working Paper, 2019, n. ${ }^{\circ}$ 152, May. PÉrez Romero, "Precios, salarios y carga laboral...", op. cit. 
En lo que respecta a la otra columna sobre la que descansa el estudio de los salarios reales, los precios y, por tanto, la construcción de un deflactor, mi propuesta de deflactor tipo se basaría en las siguientes premisas metodológicas. En primer lugar, no obstante las dificultades que puedan encontrarse en la búsqueda de series de precios más allá de las acostumbradas "subsistencias», en el estado actual de la investigación se debe afrontar el reto de construir deflactores de segunda generación ${ }^{25}$, incluyendo el vestido, el combustible y la vivienda como parte de los presupuestos familiares. Por otro lado, más allá de los índices de precios de tipo Laspeyres, debe ensayarse también el cálculo de índices geométricos (media geométrica ponderada), ya que permiten tener en cuenta adaptaciones del consumo en respuesta a cambios en los precios relativos ${ }^{26}$. En lo que respecta al establecimiento del porcentaje dedicado en las cestas de consumo a cada una de las principales partidas, la mayoría de las investigaciones centradas en la España preindustrial coinciden en suponer un gasto en alimentación comprendido entre dos tercios y tres cuartos del gasto total.

Finalmente, más allá de la medición de la capacidad de consumo en términos de subsistencias como el trigo o de un índice de precios, a efectos comparativos cabría ensayar la metodología de las ratios de bienestar (welfare ratios) y las canastas de consumo de supervivencia (barebone baskets) propuesta por Allen en su famoso artículo publicado en 2001. Sin embargo, una vía más realista y coherente con los postulados metodológicos defendidos en este trabajo pasaría por la construcción de deflactores cimentados en una "frontera de posibilidades de consumo ${ }^{27}$ en la que se consideren al menos todos los ingresos familiares por la vía del jornal, el tamaño familiar y sus cambios, el número de días trabajados y el consumo -cantidad y calidades- efectivamente realizado.

\subsection{La desigualdad económica}

Una derivada importante del estudio del bienestar material es la relacionada con la medición de la desigualdad económica. Aspecto que la historia económica no ha abordado todavía en profundidad y que solo recientemente parece haber

25. Entendiendo como deflactores de segunda generación aquellos que incluyen, al menos, partidas como el alquiler de vivienda, el combustible, el vestido y el calzado y tienen en cuenta los cambios producidos en los patrones de consumo ajustando las ponderaciones del índice o incluyendo nuevos productos. Véase, por ejemplo, Llopis, Enrique; García-Hiernaux, Alfredo; García-MonTERo, Héctor; González Mariscal, Manuel y Hernández García, Ricardo. «Índices de precios de tres ciudades españolas, 1680-1800: Palencia, Madrid y Sevilla». América Latina en la Historia Económica, 2009, 16, 2, pp. 29-80.

26. Allen, Robert C. "The Great Divergence...», op. cit., pp. 423-424.

27. Calderón Fernández, Andrés; García-Montero, Héctor y Llopis Agelán, Enrique. "New research guidelines...", op. cit. 
comenzado a adquirir cierto protagonismo en el debate académico ${ }^{28}$. En este sentido, algunos estudios han comenzado a proporcionar evidencias directas o indirectas sobre la trayectoria de la desigualdad en la renta y la riqueza en la España del Siglo de las Luces.

Cabe comenzar por señalar que, lógicamente, las distintas estimaciones de la evolución del PIB por habitante son a priori compatibles con una distribución más o menos desigual del producto e incluso, aun existiendo crecimiento económico, con un menor bienestar material en amplias capas de la población. En este sentido, la aceptación de un crecimiento económico positivo hasta finales del Setecientos choca con las impresiones y testimonios de contemporáneos mostrando una sensación de crisis económica y, más en concreto, preocupación por el endeudamiento, la inviabilidad de las pequeñas explotaciones campesinas y, en definitiva, el creciente empobrecimiento de buena parte de la sociedad; a lo que cabría añadir nuestro conocimiento sobre la trayectoria seguida por otras variables clave como los salarios reales o la renta de la tierra. Por tanto, no es de extrañar que algún autor ${ }^{29}$ haya concluido que los niveles de vida se estaban deteriorando de forma importante a finales del siglo XVIII.

Un claro descenso de los salarios reales y un incremento de la renta de la tierra $^{30}$ parecen mostrar un aumento en las últimas décadas del Setecientos de la desigualdad en la distribución factorial de la renta y el producto agrario ${ }^{31}$. La relación renta-salarios, siguiendo una dinámica ricardiana, habría aumentado hasta los primeros años del siglo XIX; como prueban las series disponibles para el sur de Navarra y el norte de Castilla ${ }^{32}$.

Mientras una minoría, cuyo mejor exponente serían los grandes labradores -e incluso medianos en función de la ratio entre tierras propias y arrendadas- y los terratenientes, se habría beneficiado de la coyuntura alcista de los precios de las

28. Véase una síntesis de las aportaciones recientes sobre el tema en ALFANI, Guido. «Wealth and Income Inequality in the Long Run of History». En DiEBOLT, Claude y HuPERT, Michael (eds.). Handbook of Cliometrics. Berlin-Heidelberg: Springer, 2019, en prensa.

29. Domínguez Martín, Rafael. "Niveles de vida e indicadores de bienestar social a finales del Antiguo Régimen: comparaciones internacionales y contrastes regionales». En LLopis, Enrique (ed.). El legado económico..., op. cit., pp. 301-327 (p. 305).

30. Las series disponibles para la España interior muestran cómo la renta alcanzó máximos, según el caso, entre 1780 y el cambio de siglo. Véase SEBASTiÁn, José Antonio. "La agricultura española...", op. cit., p. 160.

31. Yun, Bartolomé. «Ingresos, formas de distribución del producto agrario y cambio social en Castilla la Vieja y León en el siglo XVIII. En Actas del Seminario de Segovia sobre: Agricultura e Ilustración en España (14-16 de septiembre de 1988). Madrid: MAPA, 1989, pp. 481-505 (p. 495). Para ello es preciso suponer que el incremento en el número de jornales empleados en el trabajo agrario fue igual o menor que el de la superficie agraria arrendada. Dicho de otra forma, que la masa salarial agraria no creció más deprisa que la masa de las rentas agrarias, pudiendo quizás compensar el incremento de la relación entre la tasa de la renta y la salarial.

32. Véanse Lana Berasain, José Miguel. "El poder de compra...", op. cit. y Moreno Lázaro, Javier. "¿Fomentó el capitalismo...?», op. cit. 
subsistencias y la renta de la tierra y del descenso del precio relativo de los jornales ${ }^{33}$, gran parte de la sociedad rural, pequeños labradores arrendatarios y jornaleros especialmente, se habría visto impelida a la búsqueda de ingresos complementarios en un intento de paliar el progresivo empobrecimiento ${ }^{34}$.

Desde un punto de vista cuantitativo, como resultado de las mencionadas estimaciones sobre la evolución del PIB en la España preindustrial, Álvarez Nogal y Prados de la Escosura proporcionan series para dos indicadores que pueden servir como proxies para trazar la evolución de la desigualdad en la distribución de la renta. En primer lugar, la relación entre la renta de la tierra y el salario, una ratio para aproximar la relación entre las retribuciones de la propiedad de la tierra -el capital más importante en una sociedad preindustrial-y del factor trabajo, es decir, la distribución factorial de la renta, apunta una tendencia creciente que arrancaría de finales del Seiscientos y llegaría hasta el inicio del Ochocientos. Situándose a finales del siglo XVIII en niveles similares a los máximos alcanzados a finales del siglo XVI ${ }^{35}$.

Por otro lado, la ratio entre el PIB nominal por habitante y el salario nominal no cualificado, indicador conocido como índice de Williamson, una proxy para la medición de la distribución del ingreso, muestra una tendencia igualmente creciente durante todo el Setecientos, tendencia que habría arrancado en las décadas posteriores a la Peste Negra y, en el largo plazo, habría continuado hasta el inicio del Ochocientos. Como matiz a sus resultados, los autores señalan la posibilidad de que durante las últimas décadas del siglo XVIII se incrementara el número de días trabajados y, por tanto, los ingresos salariales queden subestimados y, en definitiva, el incremento de la desigualdad sobreestimado ${ }^{36}$.

33. Lo cual, aun habiendo podido jugar también un papel los cambios en los precios relativos o en las elasticidades, es coherente con el incremento del consumo de productos duraderos y semiduraderos detectado en inventarios post-mórtem y cartas de dote si tenemos en cuenta que quienes realizaron estos documentos pertenecían en un alto porcentaje al grupo de los beneficiados por la coyuntura. Véanse, por ejemplo, los trabajos incluidos en el monográfico dedicado por la Revista de Historia Económica (2003) a «El consumo en la España preindustrial». Síntesis de las aportaciones en esta línea de investigación para el caso español pueden encontrarse en BERNARDOS SANZ, José Ubaldo. "El consumo en España (1750-1850)». En Llopis, Enrique (ed.). El legado económico..., op. cit., pp. 273300 (pp. 287-289) y Domínguez Martín, Rafael. "Niveles de vida...», op. cit., p. 315.

34. Yun, Bartolomé. "Ingresos...», op. cit.; y "Mercado de cereal y burguesía en Castilla, 17501868". En Yun, Bartolomé (coord.). Estudios sobre capitalismo agrario, crédito e industria en Castilla (siglos XIX Y XX). Valladolid: Junta de Castilla y León, 1991, pp. 4 7-76 (p. 51).

35. Álvarez Nogal, Carlos y Prados de la Escosura, Leandro. "The rise and fall...", op. cit., p. 9.

36. Ibidem, pp. 20-21. Una visión a contracorriente, descubriendo una caída de la desigualdad en la distribución del ingreso a finales del Setecientos en la provincia de Guadalajara, puede encontrarse en SANTIAGO-CABALLERO, Carlos. "Income inequality in central Spain, 1690-1800». Explorations in Economic History, 2011, 48, 1, pp. 83-96. Sobre la desigualdad en el ingreso y su relación con el capital humano véase un estudio reciente, basado en datos del Catastro de Ensenada, en ÁlvarEz, Begoña y Ramos Palencia, Fernando. "Human capital and earnings in eighteenth-century Castile». Explorations in Economic History, 2018, 67, 1, pp. 105-133. 
Finalmente, algunas estimaciones recientes acerca de la evolución de la distribución de la riqueza en regiones como Cataluña o Castilla, incluso partiendo de metodologías y fuentes diversas, apuntan también claramente hacia un crecimiento de la desigualdad a lo largo del Setecientos ${ }^{37}$.

\section{INDICADORES BIOLÓGICOS}

El protagonismo adquirido en los últimos años por la historia antropométrica en la historia económica contemporánea española no ha alcanzado todavía a lo sucedido en el Antiguo Régimen. Si actualmente nos es relativamente bien conocida la trayectoria seguida por la estatura media -reconocido indicador en la literatura biomédica del estatus nutricional neto de una población- en diversas series regionales y locales desde mediados del siglo $\mathrm{XIX}^{38}$, sabemos bastante menos sobre las generaciones que crecieron durante el Setecientos y en las últimas décadas del Antiguo Régimen. Las dificultades que plantea la documentación local ${ }^{39}$, los Expedientes Generales de Reemplazo, habitualmente empleada para el estudio de épocas posteriores, y la ausencia de fuentes con cobertura nacional parecen haber sido hasta el momento un obstáculo casi insalvable para el progreso de la historiografía española.

A dicho panorama tan solo escaparían, hasta el momento, los estudios basados en los escasos Padrones de Alistamiento conservados de entre aquellos realizados en 1808 por algunas Juntas Provinciales o ayuntamientos para organizar la lucha contra la invasión napoleónica. En el caso de la antigua provincia de Toledo, la conservación de la fuente para gran parte de la provincia ha permitido, a través del estudio de los mozos alistados en 99 localidades, establecer con precisión el estatus nutricional de los nacidos entre finales de las décadas de 1760 y 1780, realizar una radiografía de sus diferencias sociales y trazar la evolución de la desigualdad biológico-nutricional ${ }^{40}$.

37. Brea-Martínez, Gabriel y Pujadas-Mora, Joana María. "Transformación y desigualdad económica en la industrialización en el área de Barcelona, 1715-1860». Revista de Historia EconómicaJournal of Iberian and Latin American Economic History, 2018, 36, 2, pp. 241-273. GARCíA-MONTERO, Héctor. "Long-term Trends in Wealth Inequality in Catalonia, 1400-1800: Initial Results». Università Commerciale Luigi Bocconi. "Carlo F. Dondena" Centre for Research on Social Dynamics (DONDENA) working papers, 2015, Working Paper 079. Nicolini, Esteban y RAMOS PALENCIA, Fernando. "Decomposing income inequality in a backward pre-industrial economy: Old Castile (Spain) in the middle of the eighteenth century». Economic History Review, 2016, 69, 3, pp. pp. 747-772.

38. Véase a este respecto MARTínez-CARrión, José Miguel. "La talla de los europeos, 1700-2000: ciclos, crecimiento y desigualdad». Investigaciones de Historia Económica, 2012, 8, 3, pp. 176-187.

39. Véase una introducción en Cámara, Antonio David. "Fuentes antropométricas en España: problemas metodológicos para los siglos XVIII y XIX". Historia Agraria, 2006, 38, abril, pp. 105-118.

40. GARCíA-MONTERO, Héctor. "Los niveles de vida...", op. cit.; Estatura y niveles de vida en la España interior, 1765-1840. Tesis doctoral no publicada. Madrid: Universidad Complutense de Madrid, 2013; "The nutritional status of manufacturing workers and craftsmen in central Spain in the eighteenth 
Partiendo de una estatura media de en torno a $163 \mathrm{~cm}$, relativamente baja pero similar o incluso superior a la encontrada en otros territorios europeos a finales del Setecientos y durante la primera mitad del Ochocientos ${ }^{41}$, tras un cierto estancamiento, las generaciones nacidas en la década de 1780 habrían comenzado a sufrir un descenso en su estatura media. Descenso que habría estado acompañado, incluso ya desde años precedentes, de un incremento en la desigualdad socioprofesional. Aquellas profesiones vinculadas con el acceso a la propiedad de la tierra, a una cierta cualificación en el sector servicios o artesanal o al acceso directo al consumo de proteínas animales habrían gozado de un estatus nutricional significativamente superior -con una brecha de casi $6 \mathrm{~cm}$ entre los hacendados y grandes propietarios y los criados y sirvientes- al de aquellas otras sin acceso a la propiedad y con baja cualificación.

En el plano internacional, la caída mostrada en el caso de la España interior no habría sido una excepción. Bien al contrario, en la Europa de finales del Setecientos la tónica fue un empeoramiento, más o menos pronunciado, del nivel de vida biológico revelado por la talla media. Por tanto, por lo que sabemos hasta el momento, el caso español no habría estado entre aquellos con un peor balance en la centuria. Lejos por ejemplo de la enorme caída sufrida por británicos o de la también importante del norte de Italia o algunas regiones francesas ${ }^{42}$.

\section{INDICADORES DEMOGRÁFICOS}

El estudio de variables relacionadas con la mortalidad, como la tasa de mortalidad bruta y, sobre todo, las tasas de mortalidad en las primeras etapas de la vida $^{43}$ y la esperanza de vida, teniendo significados y determinantes parcialmente diversos, proporciona indicadores unánimemente aceptados y habitualmente utilizados en la evaluación de las condiciones de vida de una población y en la

century». Revista de Historia Industrial, 2016, 64, monográfico 2, pp. 51-75; y "La desigualdad en el estado nutricional en la España interior a finales del siglo XVIII. Nutrición Hospitalaria, 2018, 35, n. extraordinario 5, pp. 26-30. Para el caso de la localidad granadina de Montefrío véanse CÁmARA, Antonio David. "Long-Term Trends in Height in Rural Eastern Andalucía (1750-1950)». Historia Agraria, 2009, 47, abril, pp. 45-67 y CÁMARA, Antonio David y GARCía ROMÁN, Joan. "Ciclos largos de nivel de vida biológico en España (1750-1950): propuesta metodológica y evidencias locales». Investigaciones de Historia Económica, 2010, 6, 2, pp. 95-118.

41. Martínez-CARrión, José Miguel. "La talla de los europeos...", op. cit.

42. Véanse Dobado GonzÁlez, Rafael y García-Montero, Héctor. "Colonial origins of inequality in Hispanic America? Some reflections based on new empirical evidence». Revista de Historia Económica-Journal of Iberian and Latin American Economic History, 2010, 28, 2, pp. 253-277. GARCÍAMONTERo, Héctor. Estatura y niveles de vida..., op. cit. MARTínEZ-CARrIón. "La talla de los europeos...", op. cit.

43. Como la neonatal (durante el primer mes de vida), postneonatal (entre el primer mes y el primer año), infantil (el primer año en su conjunto) o «juvenil» (del primero al cuarto año). 
elaboración de los indicadores sintéticos de bienestar construidos por las agencias internacionales de desarrollo.

En particular, la demografía histórica ha destacado el importante papel jugado por la mortalidad a edades tempranas en la caída de la mortalidad en los inicios del proceso de transición demográfica. Proceso que, en el sur de Europa, habría tenido la particularidad de una mayor mortalidad juvenil respecto a la infantil -fenómeno atribuido a unas peores condiciones de vida y unas peores estructuras higiénico-sanitarias-, pero con un comienzo en su caída -de la juvenil- más temprano y rápido, situándose por debajo solo desde comienzos del siglo $\mathrm{XX}^{44}$.

¿Qué progresos pueden constatarse en la demografía histórica española ocupada en el estudio del Setecientos desde el punto de vista de los indicadores de mortalidad mencionados? ¿Qué vías de investigación cabe sugerir para el futuro? A la vista de lo comentado en el párrafo anterior, debería ser superfluo detenerse en la importancia del estudio de la mortalidad a edades tempranas, máxime cuando la estadística oficial no proveyó con cifras -y en todo caso escasas, incompletas y discontinuas- hasta entrado el siglo XIX. Quizás por ello, sin embargo, no son muchas las investigaciones que se han aventurado en la laboriosa reconstrucción de series a partir de la documentación conservada en los archivos parroquiales; insumo básico de los estudios demográficos sobre la España del Antiguo Régimen.

En los últimos años, investigadores vinculados al Grupo Complutense de Historia Económica Moderna ${ }^{45}$ han acometido la ímproba tarea de trazar la evolución de la mortalidad en diversos territorios de la España interior rural desde 1700 hasta las postrimerías del siglo XIX, a través de numerosas muestras de localidades seleccionadas entre multitud de archivos parroquiales. Los resultados revelan una caída secular de la tasa de mortalidad bruta ya desde mediados del siglo XVIII. Un

44. PÉrez MoredA, Vicente; Reher, David-Sven y SANZ GIMENO, Alberto. La conquista de la salud. Mortalidad y modernización en la España contemporánea. Madrid: Marcial Pons, 2015 (p. 149).

45. Véanse ABARCA, Vanesa y LANZA, Ramón. "El declive de la mortalidad en el interior castellano y la cornisa cantábrica, 1700-1860: un estudio comparado”. Comunicación presentada al XIV Congreso de la Sociedad Española de Historia Agraria. Badajoz, 2013. Abarca, Vanesa; Sebastián, José Antonio; LLOPIS, Enrique; BERnARDOS, José Ubaldo y VELASCO, Ángel Luis. «El descenso de la mortalidad en la España interior: Albacete y Ciudad Real, 1700-1895”. América Latina en la Historia Económica, 2015, 22, 3, pp. 108-144. Abarca, Vanesa; Llopis, Enrique; SalaZar, Felipa y Velasco, Ángel Luis. "El descenso de la mortalidad en la España interior: Albacete y Ciudad Real, 1700-1895". Revista Uruguaya de Historia Económica, 2016, VI, 9, pp. 9-30. Llopis, Enrique; BERnARdos, José Ubaldo y Velasco, Ángel Luis. "¿Pasó de largo por la España interior la primera fase de la transición demográfica? La mortalidad en Ávila y Guadalajara, 1700-1895”. Investigaciones de Historia Económica, 2015, 11, 2, pp. 69-79. LLOPIS, Enrique; Alonso, Elvira; Fontanilla, Paloma; Hípola, Belén; Méndez, Sara y Ramos, Javier. "La mortalidad cerca de una gran ciudad: el mundo rural madrileño en los siglos XVIII y XIX". Comunicación presentada al XII Congreso de la AEHE. Salamanca, 2017a. LlOPIS, Enrique; ALONSO, Elvira; FONTANILLA, Paloma; Hípola, Belén; MÉNDEZ, Sara y RAmos, Javier. "Crecimiento económico sin mayor bienestar. Mortalidad, mortalidad catastrófica e inestabilidad demográfica y económica en Murcia, 1769-1895”. Comunicación presentada al XII Congreso de la AEHE. Salamanca, 2017b. 
proceso que habría sufrido interrupciones, las más claras en los primeros años del Ochocientos y entre 1860 y 1890, y que habría sido guiado por el descenso de la mortalidad ordinaria. Por otro lado, la mortalidad adulta habría sido protagonista frente a la resistencia a la baja o la menor caída de la mortalidad a edades tempranas o parvularia ${ }^{46}$.

Por otro lado, cabe mencionar los trabajos de Ramiro y Sanz para la España central (provincias de Madrid y Toledo y localidades de Guadalajara y Extremadura ${ }^{47}$. En ellos se trazó la evolución de la mortalidad desde finales del siglo XVIII o principios del XIX, desde el nacimiento hasta los 10 años, distinguiendo entre aniversarios -e incluso meses en el primer año-, sexo y causas de defunción.

Asimismo, merecen especial atención las investigaciones llevadas a cabo para otros territorios peninsulares abarcando en parte o en su totalidad el siglo XVIII, casos de Vizcaya ${ }^{48}$, Mallorca ${ }^{49}$ o algunas comarcas gerundenses ${ }^{50}$. Trabajos que han apuntado también a transformaciones relativamente tempranas en los patrones y los niveles de mortalidad mostrando, en definitiva, un panorama menos sombrío del tradicionalmente aceptado sobre la evolución de la mortalidad y la transición demográfica en la España de los siglos XVIII y XIX.

¿Por dónde podrían transcurrir los derroteros de la investigación en los próximos años? Como puede deducirse de la historiografía mencionada, es mucho lo que todavía desconocemos acerca de las primeras etapas de la caída secular de la mortalidad; en un mapa peninsular que, por otro lado, dista mucho todavía de poder ofrecer una visión general mínimamente representativa y completa.

En concreto, más allá de la extensión de la base empírica a otros territorios, tanto rurales como urbanos, me atrevo a sugerir dos vías por las que la investigación podría contribuir a un mejor conocimiento en los aspectos y debates señalados. En primer lugar, las series de mortalidad a edades tempranas podrían

46. Una visión diferente acerca de las causas del proceso puede encontrarse en PÉREz ROMERO, Emilio. "¿Por qué disminuyó la mortalidad en la España interior entre 1700 y 1850?: El caso de la provincia de Soria». En Comín, Francisco; Hernández García, Ricardo y Moreno, Javier (coords.). Instituciones políticas, comportamientos sociales y atraso económico en España (1580-2000): Homenaje a Ángel García Sanz. Salamanca: Ediciones de la Universidad de Salamanca, 2017, pp. 55-86.

47. RAMIRO FARIÑAS, Diego. La evolución de la mortalidad en la infancia en la España interior, 1785-1960. Tesis doctoral inédita. Madrid: Universidad Complutense de Madrid, 1998. San Gimeno, Alberto y RAMIRO FARIÑAS, Diego. "Infancia, mortalidad y niveles de vida en la España interior, siglos XIX y xX». En Martínez-CARrión, José Miguel (coord.). El nivel de vida..., op. cit., pp. 359-404.

48. Guerrero, Ana; Arbaiza, Mercedes y PAreja, Arantza. «Mundo rural y mundo urbano en la transición de la mortalidad vizcaína (1770-1930)». Boletín de la Asociación de Demografía Histórica, 1996, XIV, 2, pp. 19-55.

49. Bujosa, Francisco; Moll, Isabel y Sureda, Bernat. "La avanzada transición demográfica en Mallorca: el caso de la mortalidad infantil». Revista de Demografía Histórica, 2000, 18, 2, pp. 125-146.

50. FERRER, Llorenc. «El crecimiento demográfico de las comarcas de Girona en el siglo XVIII y los cambios hacia la transición demográfica en la primera mitad del siglo XIX”. Revista de Demografía Histórica, 2016, 34, 2, pp. 17-52. 
retrotraerse hasta el siglo XVIII. Ciertamente son muy pocos los registros parroquiales que disponen de información de calidad para dicha centuria, pero, como es conocido y revelan, entre otros mencionados, los estudios del Grupo Complutense, no por ello inexistentes. Por tanto, aun con muestras regionales relativamente pequeñas, el ejercicio podría ser muy revelador. Mayores dificultades metodológicas plantea -incluso ya para el siglo XIX- el estudio de las causas de defunción, pero ello no debe ser obstáculo para intentar avanzar en su estudio. Incluso con una base empírica reducida, trabajando con grandes tipos de causas de defunción y, sin duda, con mayores márgenes de error, los avances en este sentido podrían aportar nuevos ingredientes y coadyuvar en la búsqueda de explicaciones acerca de la evolución en el largo plazo de la mortalidad y sus causas.

\section{CONCLUSIONES}

Este trabajo ha planteado un estado general de la cuestión en lo que atañe a los trabajos ocupados del estudio de los niveles de vida en la España del Setecientos. En concreto se ha centrado en tres grupos de indicadores, aquellos ocupados del bienestar material como el PIB por habitante o los salarios reales, los biológico-nutricionales, caso de la estatura media, y los demográficos, fundamentalmente los centrados en la medición de la mortalidad.

Aunque el debate permanece abierto, todo parece indicar que la economía española fue capaz de incrementar ligeramente el bienestar material por habitante durante el Siglo de las Luces. Menos optimismo aporta, sin embargo, el hecho de que indicadores como los salarios reales muestren una caída acusada en las décadas finales de la centuria. Si bien existe cierto debate acerca de si la caída en dicho tipo de ingresos pudo ser compensada por otros elementos de la ecuación de los ingresos familiares, entre los que cabría una suerte de "revolución industriosa " ${ }^{51}$, todo parece apuntar a que la centuria se cerró con un incremento significativo de la desigualdad económica. Aunque este aspecto merece mucha más atención de la que ha concitado hasta el momento, la trayectoria de la ratio entre la renta de la tierra y el salario no cualificado, del cociente entre el salario y el PIB nominal por habitante y el salario nominal no cualificado (índice de Williamson) o algunas estimaciones acerca de la evolución de la distribución de la riqueza en regiones como Cataluña o Castilla señalan unánimemente en este sentido.

La carencia hasta el momento de series de estatura media que arranquen de la primera mitad del siglo impide vislumbrar la trayectoria seguida por el estatus nutricional neto de los españoles durante buena parte de la centuria. Sin embargo, la evidencia disponible para el interior peninsular y alguna localidad andaluza,

51. VRIES, Jan de. The Industious Revolution: Consumer Behavior and the Household Economy, 1650 to the Present. Cambridge: Cambridge University Press, 2008. 
descubre una caída para los nacidos en las últimas décadas, importantes diferencias sociales y un incremento significativo de la desigualdad en la coyuntura de las décadas finales del Setecientos.

Finalmente, por lo que atañe a los indicadores de mortalidad, las investigaciones más recientes han detectado un patrón de caída de la mortalidad, sobre todo la ordinaria y adulta, tanto en la España interior como en algunas áreas de la periferia peninsular. Lo cual reforzaría el panorama moderadamente optimista encontrado en algunos indicadores materiales y sugeriría un comienzo de la transición demográfica más temprano, aunque probablemente más lento y accidentado que en otros países de Europa occidental, de lo que tradicionalmente se ha venido señalando.

\section{Bibliografía}

Abarca Abarca, Vanesa y LanZa García, Ramón. «El declive de la mortalidad en el interior castellano y la cornisa cantábrica, 1700-1860: un estudio comparado". Comunicación presentada al XIV Congreso de la Sociedad Española de Historia Agraria. Badajoz, 2013.

Abarca Abarca, Vanesa; Llopis Agelán, Enrique; Salazar, Felipa y Velasco Sánchez, Ángel Luis. «El descenso de la mortalidad en la España interior: Albacete y Ciudad Real, 17001895". Revista Uruguaya de Historia Económica, 2016, VI, 9, pp. 9-30.

abarca Abarca, Vanesa; Sebastián Amarilla, José Antonio; llopis Agelán, Enrique; BerNARDOS, José Ubaldo y VELASCO SÁNCHEZ, Ángel Luis. "El descenso de la mortalidad en la España interior: Albacete y Ciudad Real, 1700-1895". América Latina en la Historia Económica, 2015, 22, 3, pp. 108-144.

AlfanI, Guido. "Wealth and Income Inequality in the Long Run of History». En Diebolt, Claude y HUPERT, Michael (eds.). Handbook of Cliometrics. Berlin-Heidelberg: Springer, 2019, en prensa.

Allen, Robert C. «Economic structure and agriculture productivity in Europe, 1300-1800». European Review of Economic History, 2000, 3, 2, pp. 1-25.

Allen, Robert C. "The Great Divergence in European Wages and Prices from the Middle Ages to the First World War». Explorations in Economic History, 2001, 38, 4, pp. 411-447.

Álvarez, Begoña y Ramos Palencia, Fernando. «Human capital and earnings in eighteenthcentury Castile». Explorations in Economic History, 2018, 67, 1, pp. 105-133.

Álvarez-Nogal, Carlos y Prados de la Escodura, Leandro. «The decline of Spain (1500-1850): conjectural estimates». European Review of Economic History, 2007, 11, 3, pp. 319-366.

Álvarez-Nogal, Carlos y Prados De la Escodura, Leandro. "The rise and fall of Spain (12701850)». Economic History Review, 2013, 66, 1, pp. 1-37.

Álvarez-Nogal, Carlos; Prados de la Escodura, Leandro y Santiago-Caballero, Carlos. "Spanish agriculture in the little divergence». European Review of Economic History, 2016, 20, 4, pp. 452-477.

ANes Álvarez, Gonzalo. Las crisis agrarias en la España moderna. Madrid: Taurus, 1970.

Beltrán TAPIA, Francisco. "Commons and the standard of living debate in Spain". Cliometrica, 2015, 9, 1, pp. 27-48. 
Bernardos Sanz, José Ubaldo. "El consumo en España (1750-1850)». En Llopis Agelán, Enrique (coord.). El legado económico del Antiguo Régimen en España. Barcelona: Crítica, 2004, pp. 273-300.

BORRAS LLOP, José María. «Una historia recuperada. Las aportaciones de la infancia al crecimiento económico y a la subsistencia familiar». En Borrás Llop, José María (coord.). El trabajo infantil en España (1750-1950). Barcelona: Icaria y Publicaciones y Edicions de la Universitat de Barcelona, 2013, pp. 9-26.

Brea-Martínez, Gabriel y Pujadas-Mora, Joana María. «Transformación y desigualdad económica en la industrialización en el área de Barcelona, 1715-1860». Revista de Historia Económica-Journal of Iberian and Latin American Economic History, 2018, 36, 2, pp. 241-273.

Bringas GuTIÉRReZ, Miguel Ángel. La productividad de los factores en la agricultura española (1752-1935). Madrid: Banco de España, 2000.

BroAdBerRy, Stephen; CAMPBell, Bruce; KleIn, Alexander; OverTon, Mark y LeEuwen, Bas van. British Economic Growth 1270-1850. Cambridge: Cambridge University Press, 2015.

Bujosa, Francisco; Moll, Isabel y SuREDA, Bernat. «La avanzada transición demográfica en Mallorca: el caso de la mortalidad infantil». Revista de Demografía Histórica, 2000, 18, 2, pp. $125-146$.

Calderón Fernández, Andrés; García-Montero, Héctor y Llopis Agelán, Enrique. "New research guidelines for living standards, consumer baskets, and prices in Madrid and Mexico». En Nigro, Gianpiero (ed.). I prezzi delle cose nell'eta preindustriale XLVIIISettimana di Studi Istituto Internazionale di Storia Economica «F. Datini». Firenze: Firenze University Press, 2017, pp 333-364.

CÁmara Hueso, Antonio David. «Fuentes antropométricas en España: problemas metodológicos para los siglos XVIII y XIX”. Historia Agraria, 2006, 38, abril, pp. 105-118.

CÁmara Hueso, Antonio David. «Long-Term Trends in Height in Rural Eastern Andalucía (1750-1950)». Historia Agraria, 2009, 47, abril, pp. 45-67.

Cámara Hueso, Antonio David y García Román, Joan. "Ciclos largos de nivel de vida biológico en España (1750-1950): propuesta metodológica y evidencias locales». Investigaciones de Historia Económica, 2010, 6, 2, pp. 95-118.

CARreras, Albert. "Modern Spain». En MOKYr, Joel (ed.). The Oxford Encyclopedia of Economic History. 5 vols. New York: Oxford University Press, 2003, 4, pp. 546-553.

Dobado González, Rafael y García-Montero, Héctor. «Colonial origins of inequality in Hispanic America? Some reflections based on new empirical evidence». Revista de Historia Económica-Journal of Iberian and Latin American Economic History, 2010, 28, 2, pp. 253-277.

Domínguez MarTín, Rafael. "Niveles de vida e indicadores de bienestar social a finales del Antiguo Régimen: comparaciones internacionales y contrastes regionales». En LLOPIS Agelán, Enrique (ed.). El legado económico del Antiguo Régimen en España. Barcelona: Crítica, 2004, pp. 301-327.

FELIU I MONFORT, Gaspar. «Precios y Salarios en la Cataluña Moderna. Volumen I: alimentos». Estudios de Historia Económica, 21, 1991a.

Feliu I MONFORT, Gaspar. "Precios y Salarios en la Cataluña Moderna. Volumen II: Combustibles, productos manufacturados y salarios». Estudios de Historia Económica, 22, $1991 b$ 
FELIU I MONFORT, Gaspar. "Aproximació a un índex del cost de la vida a Barcelona, 15011807». En Fontana, Josep (ed.). Història i projecte social. Reconeixement a una trajèctoria. Barcelona: Crítica, 2004, pp. 151-170.

FERRER I ALÒs, Llorenç. "El crecimiento demográfico de las comarcas de Girona en el siglo XVIII y los cambios hacia la transición demográfica en la primera mitad del siglo XIX». Revista de Demografía Histórica, 2016, 34, 2, pp. 17-52.

GarcíA-Montero, Héctor. Los niveles de vida en la España del Antiguo Régimen. Estado de la cuestión y propuestas de investigación. En CHASTAGNARET, Gerard; DAumas, JeanClaude; Escudero, Antonio y Raveux, Olivier (eds.). Los niveles de vida en España y Francia (siglos XVIIII-XX). Alicante: Publicaciones de la Universidad de Alicante-Publications de la Universitè de Provence, 2010, pp. 21-44.

GARCíA-MONTERO, Héctor. Estatura y niveles de vida en la España interior, 1765-1840. Tesis doctoral no publicada. Madrid: Universidad Complutense de Madrid, 2013.

GARCíA-MONTERo, Héctor. «Long-term Trends in Wealth Inequality in Catalonia, 1400-1800: Initial Results". Università Commerciale Luigi Bocconi. "Carlo F. Dondena" Centre for Research on Social Dynamics (DONDENA) working papers, 2015, Working Paper 079.

GARCíA-MONTERO, Héctor. "The nutritional status of manufacturing workers and craftsmen in central Spain in the eighteenth century». Revista de Historia Industrial, 2016, 64, monográfico 2 , pp. 51-75.

GARCíA-MonTERO, Héctor. "La desigualdad en el estado nutricional en la España interior a finales del siglo XVIII". Nutrición Hospitalaria, 2018, 35, número extraordinario 5, pp. 26-30.

GARCíA-MonTERo, Héctor. «Historia agraria y niveles de vida. Estado de la cuestión y propuestas de investigación para la España contemporánea». En SOTO FERNÁNDEZ, David y LANa Berasáin, José Miguel (eds.). Del pasado al futuro como problema. La historia agraria contemporánea española en el siglo XXI. Zaragoza: Prensas Universitarias de Zaragoza, 2019, pp. 125-144.

GARCía ZÚÑIGA, Mario. "Fêtes chômées et temps de travail en Espagne (1250-1900)». En MatTTE, Corine y TerRIER, Didier (dir.). Les temps du travail. Normes, pratiques, évolutions (XIVe-XIXe siècles). Rennes: Presses Universitaires de Rennes, 2014, pp. 63-80.

García-ZúÑIga, Mario y LóPEZ-LosA, Ernesto. "Building Workers in Madrid (1737-1805). New Wage Series and Working Lives». EHES Working Paper, 2019, May, n. ${ }^{\circ} 152$.

GaRRABOU, Ramón y TelLo, Enric. "Salario como coste, salario como ingreso: el precio de los jornales agrícolas en la Cataluña contemporánea, 1727-1930». En MARTínez CARRIÓN, José Miguel (coord.). El nivel de vida en la España rural, siglos XVIII-XX. Alicante: Publicaciones de la Universidad de Alicante, 2002, pp. 113-182.

Guerrero, Ana; ArbaizA, Mercedes y PAReja, Arantza. "Mundo rural y mundo urbano en la transición de la mortalidad vizcaína (1770-1930)». Boletín de la Asociación de Demografía Histórica, 1996, XIV, 2, pp. 19-55.

HERNÁNDEZ García, Ricardo. "El trabajo en la industria textil de Tierra de Campos a mediados del siglo XVIII: la fábrica de Astudillo». En SARASÚA, Carmen y GálveZ, Lina (eds.). ¿Privilegios o eficiencia?: Mujeres y hombres en los mercados de trabajo. Alicante: Publicaciones de la Universidad de Alicante, 2003, pp. 113-135.

HERNÁNDEZ GARCíA, Ricardo. "La mano de obra infantil en la Castilla rural del siglo XVIII: El trabajo del niño es poco pero el que lo desprecia, un locom. En Borrás Llop, José María (coord.). El trabajo infantil en España (1750-1950). Barcelona: Icaria y Publicaciones y Edicions de la Universitat de Barcelona, 2013, pp. 91-116. 
JOVER AVELlà, Gabriel. "La difusión del trabajo asalariado infantil en la agricultura mallorquina: una aproximación a partir de los libros de cuentas de los predios oleícolas, 1700-1850». En BORRÁs Llop, José María (coord.). El trabajo infantil en España (17501950). Barcelona: Icaria y Publicaciones y Edicions de la Universitat de Barcelona, 2013, pp. 27-62.

JOVER AVELlà, Gabriel. «Salarios y movilización del trabajo femenino en el olivar: Mallorca, 1650-1720». En ORTEGA LóPEZ, María Teresa (ed.). Jornaleras, campesinas y agricultoras: la historia agraria desde una perspectiva de género. Zaragoza: Prensas Universitarias de Zaragoza, 2015, pp. 43-64.

LANA Berasain, José Miguel. «El poder de compra de jornaleros y criados: Salarios reales y mercados de trabajo en la Navarra rural (1781-1936)». Investigaciones de Historia Económica, 2007, 7, 1, pp. 37-68.

LLOPIS AgELÁN, Enrique. "Expansión, reformismo y obstáculos al crecimiento (1715-1789)». En Comín, Francisco; Hernández, Mauro y Llopis Agelán, Enrique (eds.). Historia Económica de España. Siglos X-XX. Barcelona: Crítica, 2002, pp. 121-164.

Llopis Agelán, Enrique; Alonso, Elvira; Fontanilla, Paloma; Hípola, Belén; Méndez, Sara y RAMOs, Javier. «La mortalidad cerca de una gran ciudad: el mundo rural madrileño en los siglos XVIII y XIX». Comunicación presentada al XII Congreso de la AEHE. Salamanca, 2017a.

Llopis Agelán, Enrique; Alonso, Elvira; Fontanilla, Paloma; Hípola, Belén; Méndez, Sara y RAMOS, Javier. "Crecimiento económico sin mayor bienestar. Mortalidad, mortalidad catastrófica e inestabilidad demográfica y económica en Murcia, 1769-1895». Comunicación presentada al XII Congreso de la AEHE. Salamanca, 2017b.

Llopis Agelán, Entique; Bernardos SANZ, José Ubaldo y Velasco SÁnchez, Ángel Luis. «¿Pasó de largo por la España interior la primera fase de la transición demográfica? La mortalidad en Ávila y Guadalajara, 1700-1895". Investigaciones de Historia Económica, 2015, 11, 2, pp. 69-79.

Llopis Agelán, Enrique y García Montero, Héctor. «Precios y salarios en Madrid, 16801800». Investigaciones de Historia Económica, 2011, 7, 2, pp. 295-309.

Llopis agelán, Enrique; García-Hiernaux, Alfredo; García-Montero, Héctor; González Mariscal, Manuel y Hernández García, Ricardo. «Índices de precios de tres ciudades españolas, 1680-1800: Palencia, Madrid y Sevilla». América Latina en la Historia Económica, 2009, 16, 2, pp. 29-80.

LóPez-Losa, Ernesto y PiQuero-Zarauz, Santiago. «Spanish real wages in the North-western mirror, 1500-1800. On the timings and magnitude of the Little Divergence in Europe». Documento de Trabajo de la AEHE, 2016, n. ${ }^{\circ}$ 16-07.

Malanima, Paolo. "The Long Decline of a Leading Economy: GDP in Central and Northern Italy, 1300-1913". European Review of Economic History, 2011, 15, pp. 169-219.

MARTín ACEÑA, Pablo. «Los precios en Europa durante los siglos XVI y XVII: estudio comparativo". Revista de Historia Económica-Journal of Iberian and Latin American Economic History, 1992, 10, 3, pp. 359-395.

MarTíneZ-Carrión, José Miguel. «El nivel de vida en la España rural, siglos XVIII-XX. Nuevos enfoques, nuevos resultados». En Martínez-Carrión, José Miguel (ed.). El nivel de vida en la España rural, siglos XVIII-XX. Alicante: Publicaciones de la Universidad de Alicante, 2002, pp. 16-74.

MarTíneZ-CARrión, José Miguel. «La talla de los europeos, 1700-2000: ciclos, crecimiento y desigualdad». Investigaciones de Historia Económica, 2012, 8, 3, pp. 176-187. 
Moreno LÁZARO, Javier. "¿Fomentó el capitalismo agrario la desigualdad? Salarios y niveles de vida en Castilla la Vieja, 1751-1861». En Martínez-Carrión, José Miguel (coord.). El nivel de vida en la España rural, siglos XVIII-XX. Alicante: Universidad de Alicante, 2002, pp. 16-74.

Nicolini, Esteban y Ramos Palencia, Fernando. "Decomposing income inequality in a backward pre-industrial economy: Old Castile (Spain) in the middle of the eighteenth century». Economic History Review, 2016, 69, 3, pp. 747-772.

PÉrez Moreda, Vicente; ReHer, David-Sven y SANZ Gimeno, Alberto. La conquista de la salud. Mortalidad y modernización en la España contemporánea. Madrid: Marcial Pons, 2015.

Pérez Romero, Emilio. "¿Por qué disminuyó la mortalidad en la España interior entre 1700 y 1850?: El caso de la provincia de Soria». En Comín, Francisco; Hernández García, Ricardo y MORENO LÁZARO, Javier (coords.). Instituciones políticas, comportamientos sociales y atraso económico en España (1580-2000): Homenaje a Ángel García Sanz. Salamanca: Ediciones de la Universidad de Salamanca, 2017, pp. 55-86.

Pérez Romero, Emilio. "Precios, salarios y carga laboral durante el siglo XVIII. Investigaciones de Historia Económica-Economic History Research. El caso de la villa de El Burgo de Osma (Soria), 2019, 15, 2, pp. 78-90.

RAMIRO FARIÑAS, Diego. La evolución de la mortalidad en la infancia en la España interior, 1785-1960. Tesis doctoral inédita. Madrid: Universidad Complutense de Madrid, 1998.

ReHer, David Sven y BAllesteros Doncel, Esmeralda. "Precios y salarios en Castilla la Nueva: la construcción de un índice de salarios reales, 1501-1991». Revista de Historia Económica-Journal of Iberian and Latin American Economic History, 1993, 11, 1, pp. 101-151.

Ringrose, David. España, 1700-1900: el mito del fracaso. Madrid: Alianza Universidad, 1996.

SANTIAGO-CABAlLERO, Carlos. "Income inequality in central Spain, 1690-1800». Explorations in Economic History, 2011, 48, 1, pp. 83-96.

SANZ GIMENO, Alberto y RAMIRO FARIÑAS, Diego. "Infancia, mortalidad y niveles de vida en la España interior, siglos XIX y XX». En MARTíneZ-CARRIÓN, José Miguel (coord.). El nivel de vida en la España rural, siglos XVIII-XX. Alicante: Universidad de Alicante, 2002, pp. 359-404.

SARASÚA, Carmen. "La industria del encaje en el campo de Calatrava». Arenal: Revista de Historia de las Mujeres, 1995, 2, 2, pp. 151-174.

SARASÚA, Carmen. "El oficio más molesto, más duro: el trabajo de las lavanderas en la España de los siglos XVIII al XX». Historia Social, 2003, 45, pp. 53-78.

SARASÚA, Carmen. "¿Activos desde cuándo? La edad de acceso al mercado de trabajo en la España del siglo XVIII». En BORRÁs Llop, José María (coord.). El trabajo infantil en España (1750-1950). Barcelona: Icaria y Publicaciones y Edicions de la Universitat de Barcelona, 2013, pp. 63-90.

SARASÚA, Carmen. "Women's work and structural change: occupational structure in eighteenth-century Spain». Economic History Review, 2019, 72, 2, pp. 481-509.

Sebastián, José Antonio. "La agricultura española y el legado del Antiguo Régimen». En Llopis Agelán, Enrique (ed.). El legado económico del Antiguo Régimen en España. Barcelona: Crítica, 2004, pp. 147-186.

VAN ZANDEN, Jan Luiten. "Wages and the standard of living in Europe, 1500-1800». European Review of Economic History, 1999, 3, 2, pp. 175-197. 
VAN ZANDEN, Jan Luiten. «Una estimación del crecimiento económico en la Edad Moderna». Investigaciones de Historia Económica, 2005, 1, 2, pp. 9-38.

VAN Zanden, Jan Luiten y VAN LeEuwen, Bas. "Persistent but not consistent: the growth of national income in Holland, 1347-1807». Explorations in Economic History, 2012, 49, 2, pp. 119-130.

VRIES, Jan de. The Industious Revolution: Consumer Behavior and the Household Economy, 1650 to the Present. Cambridge: Cambridge University Press, 2008.

Yun, Bartolomé. «Ingresos, formas de distribución del producto agrario y cambio social en Castilla la Vieja y León en el siglo XVIII». En Actas del Seminario de Segovia sobre: Agricultura e Ilustración en España (14-16 de septiembre de 1988). Madrid: MAPA, 1989, pp. 481-505.

Yun, Bartolomé. «Mercado de cereal y burguesía en Castilla, 1750-1868». En Yun, Bartolomé (coord.). Estudios sobre capitalismo agrario, crédito e industria en Castilla (siglos XIX $y$ XX). Valladolid: Junta de Castilla y León, 1991, pp. 47-76.

Yun, Bartolomé. «Proposals to quantify long-term performance in the Kingdom of Castile, 1550-1800». En Maddison, Angus y VAN DeR Wee, Herman (eds.). Economic Growth and Structural Change: Comparative Approaches over the Long Run. Milan: Università Bocconi, 1994, pp. 97-110.

Yun, Bartolomé. «Introducción». En Yun, Bartolomé y TORRAs Elías, Jaume (dirs.). Consumo, condiciones de vida y comercialización: Cataluña, Castilla, siglos XVII-XIX. Valladolid: Consejería de Educación y Cultura-Junta de Castilla y León, 1999, pp. 1-26. 